\title{
Michael Rossmann, data-sharing champion
}

\author{
We pay tribute to an extraordinary structural biologist who was also an early advocate for data sharing.
}

L ike so many others in our community, we were saddened to learn about Michael Rossmann's death last May. In an Obituary in this issue, Hao Wu and Eddy Arnold commemorate Michael's lifelong passion for science and his many key, seminal contributions to structural biology (https:// doi.org/10.1038/s41594-019-0271-5). We often interacted with Michael as a valued author and reviewer, and we met with him at various conferences. Michael was certainly opinionated and had no qualms about sharing his views. His straightforward manner could sometimes be startling, but he was never mean-spirited or cruel. In fact, we came to appreciate that Michael was a very generous person and scientist who cared deeply about his mentees and was proud of their accomplishments. We will miss him dearly.

Here we wish to celebrate Michael's steadfast support for open science and data sharing. It might seem strange nowadays, but sharing atomic coordinates was not a standard procedure among structural biologists until the 1990s, even though the PDB was established in 1971. Helen Berman recalls that "Michael was a very early proponent of the PDB and worked hard to get people to deposit data. In the early 1970s, he sent me lists of protein crystallographers we could contact to get them to submit data." In 1978, as members of the editorial board at the Journal of Biological Chemistry, Michael and fellow structural biologist Martha Ludwig were responsible for a policy that strongly encouraged the deposition of coordinates for publication at that journal. This stance by the journal was far ahead of the curve, preceding Fred Richards' and Dick Dickerson's efforts to rally the community, which culminated with the publication of the International Union of Crystallography guidelines in 1989 (Acta Crystallogr. A 45, $658 ; 1989)$. Over the next few years, journals and funding agencies adopted requirements for structural data deposition. The heated debate among the community at the time of that transition was vividly captured in a 1989 news piece by Marcia Marinaga in Science (Science 245, 1179-1181; 1989).

During that period, annoyed by the unavailability of coordinates for published structures, Michael developed and published, together with Patrick Argos, a computer program that extracted such information from the stereo figures that often graced articles (Acta Crystallogr. B 36, $819-823 ; 1980)$. In their own article, the ethics question was discussed as follows: "The tradition of science is to gather and publish facts....The trend to withhold coordinates appears to be at odd with this long-standing tradition of scientific endeavor and exchange."

We fully concur, and it is encouraging to see how far data-sharing practices have evolved since then. Journals and funding agencies now actively engage with the research communities to discuss what type of data should be shared and how this should be achieved. New repositories for primary structural biology data have been developed, such as SBDG (for X-ray diffraction images) and EMPIAR (for EM images), and the BMRB has also expanded the types of NMR data they can host; we hope that funding agencies will continue to support these initiatives. These developments reflect a change in the mindset of structural biologists, and we honor Michael Rossmann for always pushing his community to do the right thing.

Published online: 5 August 2019

https://doi.org/10.1038/s41594-019-0282-2

\section{Watching the daisies grow}

\author{
On 15 July 2019, the Governor of the Bank of England announced that English mathematician, computer scientist \\ and cryptanalyst Alan Turing will be the new face of the $£ 50$ note.
}

W e are delighted by the recognition of Turing's life and work. He was instrumental to the development of theoretical computer science, and his mathematical model of an abstract machine that manipulates symbols on a strip of tape according to a table of rules - the so-called 'Turing machine' - can be considered an archetype of a general-purpose computer. During World War II, Turing played a pivotal role in breaking the German Enigma ciphers, a scientific triumph that was critical to the Allied victory.

Perhaps less widely known is Turing's interest in mathematical biology, although his work on the development of patterns and shapes in nature is considered seminal. In his article "The Chemical Basis of Morphogenesis," published in 1952, he suggested that a system of chemicals reacting with each other and diffusing across space, called a 'reaction-diffusion system', could account for "the main phenomena of morphogenesis." Although at times controversial, especially among experimental biologists, and not applicable to every system, it has served as a key model in theoretical biology.

The mechanisms underlying organization in biological systems that so fascinated Turing are also a topic of interest to us at Nature Structural \& Molecular Biology. Progress in structural biology has allowed the capture of ever-sharper snapshots of proteins, nucleic acids and complexes thereof, but the full picture of how those molecules act in the cell and how they influence higher-order tissue organization is still blurry. How are molecules organized within the cell, and how did they achieve such organization? How is diversity generated from uniform systems, both on the cellular level and the organismal level? How do biological systems respond to environmental signals?
With the advent of single-cell sequencing and imaging technologies, the principles behind the mechanisms of biological organization can now be addressed and understood at new depths. The development of fluorescent probes for tagging multiple proteins or sensors for the detection of specific enzymatic activities in live cells is a welcome addition to the cell biologist's tool belt. We are excited that the community is now poised to explore those long-standing questions and work toward closing the 'mesoscale gap', which will require the integration of multiple approaches and multidisciplinary collaboration. We look forward to seeing those studies come to light, in our pages and elsewhere.

Published online: 5 August 2019 https://doi.org/10.1038/s41594-019-0281-3 